\title{
Reconstruction of 3D Surface Temperature from IR images
}

by G. Cardone and S. Discetti*

*Università di Napoli Federico II, Dipartimento di Ingegneria Aerospaziale , Napoli, Italy

\section{Abstract}

The aim of the present work is to develop a camera calibration technique for high-accuracy $3 \mathrm{D}$ vision metrology using IR thermal imagers. The final task is the reconstruction of 3D surface temperature from IR images (and, as a consequence, thermal fluxes) in wind tunnels. Particular attention is given to the application in hypersonic wind tunnel "Scirocco" (CIRA).

The proposed camera calibration technique, based on the model introduced by R.Y. Tsai [1], is able to individuate the correspondence between " $3 \mathrm{D}$ camera" coordinate system and $2 \mathrm{D}$ "thermal image" coordinate system. This reconstruction procedure is very useful for test articles with three-dimensional geometry and in the presence of elaborated optical path (e. g., in case of use of mirrors to measure temperature nearby stagnation points).

The matching is determined by a set of parameters, regarding the characteristics of the camera system and the condition of shooting of the camera. The process of calibration implies the acquisition of three IR images of a suitable target. A developed calibration software detects the position in the image coordinate system of a set of markers, disposed on target's surface and finds the calibration parameters through a regression process.

A plane target with hot markers was realized with the aim of being used for the acquisition of calibration images in Scirocco. It is provided with a coupling flange, in order to allow its placement in 16 different configurations, and to allow its mounting on the Scirocco model support system. (see fig. 1)

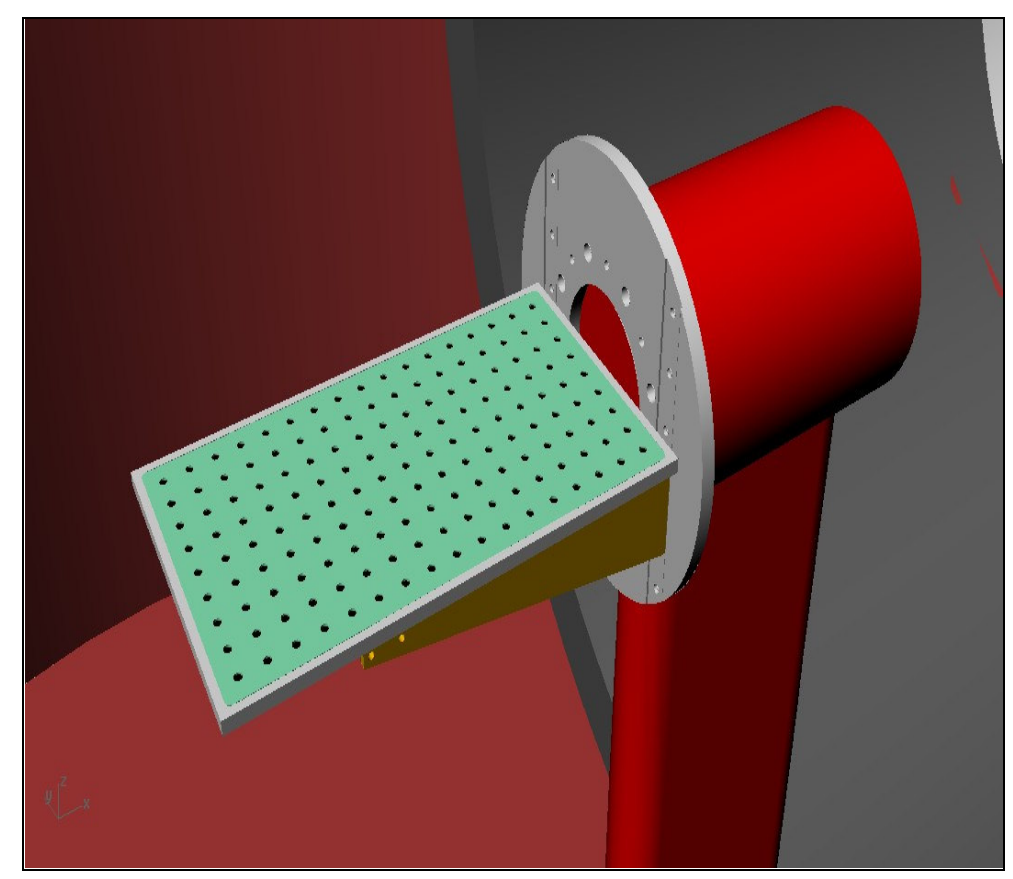

Fig. 1. IR Calibration target 
The reconstruction of $3 D$ thermal map from IR thermal images is based on the localization of a set of points disposed on test article's surface (model). A 3D virtual model of Scirocco's test chamber (fig. 2a) was implemented in order to be used as a reference system and to optimize the condition of shooting in order to obtain the lowest viewing angle for the model stagnation point using a IR mirror as shown in fig. $2 \mathrm{~b}$
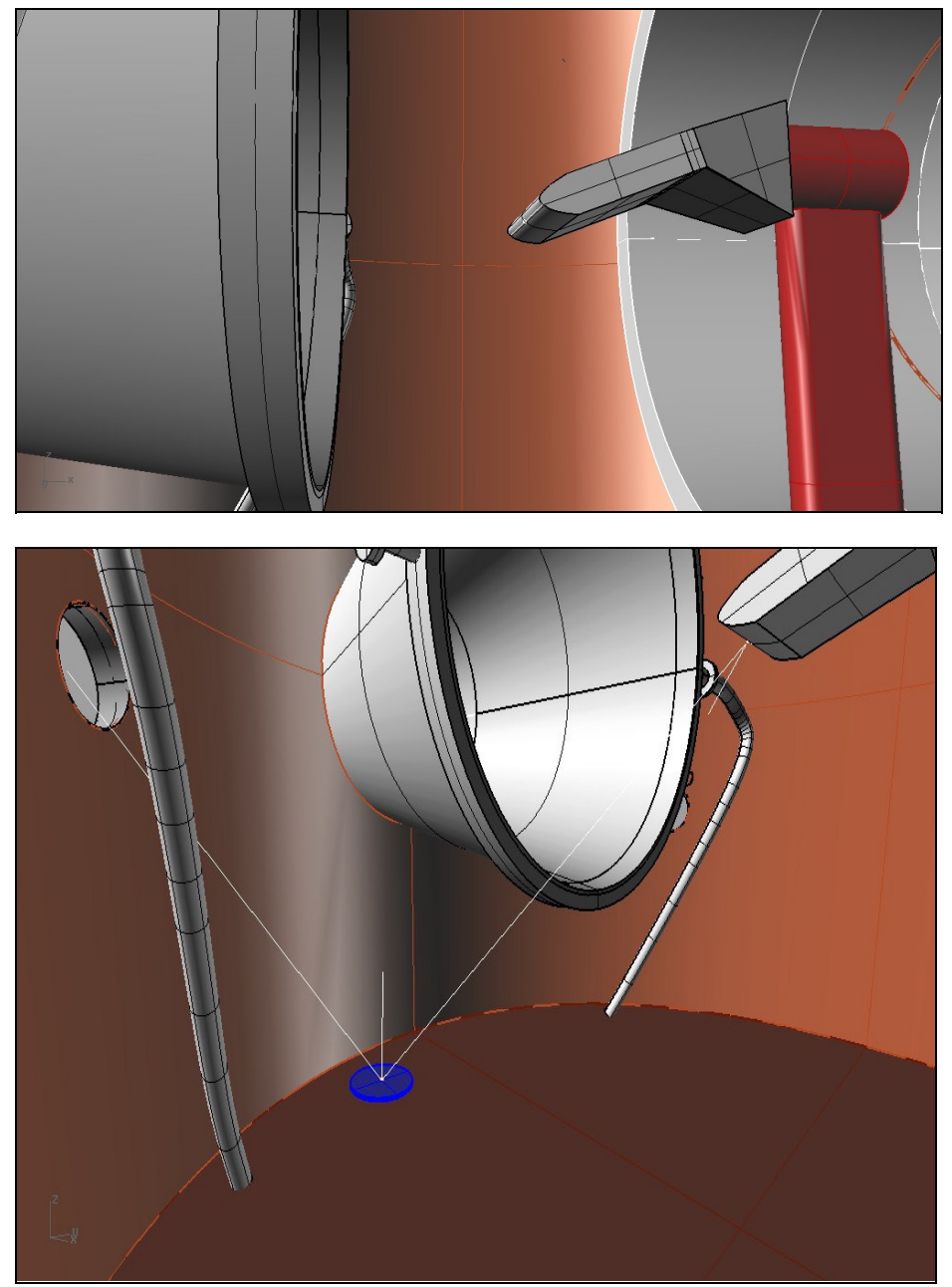

Fig. 2 - a) 3D virtual model of Scirocco's test chamber; b) IR Mirror and stagnation point optical path

The individuation of the corresponding image points is determined with Tsai's model; the local temperature is calculated by bi-linear interpolation of the values of grey level of thermal image in the adjacent pixels and the emissivity value corresponding to the corresponding view angle.

The surface of the model is then reconstructed in a tridimensional reference system, obtaining a tridimensional thermal map; the evaluation of temperature in points with known position in the physical space makes possible the reconstructions of thermal fluxes over 3D models by means of heat transfer inverse methods [2].

\section{References}

[1] R. Y. Tsai, An Efficient and Accurate Camera Calibration Technique for $3 D$ Machine Vision. Proceedings of IEEE Conference on Computer Vision and Pattern Recognition, Miami Beach, FL, (1986) pp. 364-374,

[2] G. Cardone, IR heat transfer measurements in hypersonic plasma flows, QIRT journal vol.4/2 (2007) 233-251 